\title{
Reply to M. Løberg et al.
}

Simer Jit Bains, University of Oslo and Akershus University Hospital

Milada Mahic, Norwegian Institute of Public Health

Tor Åge Myklebust, Cancer Registry of Norway

Milada Cvancarova Småstuen, University of Oslo

Sheraz Yaqub, Oslo University Hospital

Liv Marit Dørum, Cancer Registry of Norway

Bjørn Atle Bjørnbeth, Oslo University Hospital

Bjørn Møller, Cancer Registry of Norway

Kristoffer Watten Brudvik, Oslo University Hospital

Kjetil Taskén, University of Oslo and Oslo University Hospital

Corresponding author: Kjetil Taskén, Centre for Molecular Medicine Norway, Nordic EMBL Partnership, University of Oslo and Oslo

University Hospital; email: Kjetil.tasken@ncmm.uio.no

Løberg et al. comment on our paper [1], where we reported on the association of aspirin use with lower risk of mortality in 23,162 Norwegian patients with colorectal cancer (CRC), of which 6,102 were regular aspirin users post-diagnosis ( $>6$ months use). With a median observation time of 3 years after diagnosis, the hazard ratio (HR) from multivariate Coxregression analyses was 0.85 and 0.95 for CRC-specific survival (CSS) and overall survival (OS), respectively.

Løberg et al. [2] focus their criticism on a sub-analysis in our report where we stratified on aspirin use before and after diagnosis of CRC and only after, and cite our finding that in the group that did not use aspirin before CRC diagnosis the HR for aspirin use was 1.0. However, this group was comparably small (1,711 patients that only used aspirin after diagnosis versus 4,391 patents that used aspirin both before and after diagnosis) and with greater uncertainty in the corresponding effect estimate (HR 1.00 (95\% CI 0.87-1.14) versus HR 0.77 (CI 0.71- 
0.84)). Furthermore, 2,354 non-users were exposed to aspirin before diagnosis, which may serve to dilute the findings.

We used multivariate Cox-regression analysis where patients dying of other causes were censored, allowing for estimation of interpretable cause-specific hazard ratios while adjusting for confounding factors. The results show that at any point after diagnosis and given the fact that the patients are still alive, the instantaneous risk of dying of colorectal cancer is $15 \%$ lower for regular aspirin users compared to non-aspirin users. Estimating cause-specific HRs is a valid and correct approach even in the presence of competing risks [3], and is in our opinion the best way to answer the research question. Estimating Fine-Gray regressions as suggested by Løberg et al. are appropriate if the aim is to compare cumulative incidences, but is not a good approach when comparing risk between groups since such models would incorporate, rather than account for, the potential differences in risk of cardiovascular deaths between aspirin users and non-users. The presence of competing risks may, as stated [1], question the validity of estimated survival curves, as such curves are only interpretable under the assumption of independence between competing causes after conditioning on other covariates. The assumption of independence is untestable but we believe it is reasonable in our material.

We find the alternative interpretations of our data by Løberg et al. to be speculative. The assumption that the majority of deceased CRC patients that used aspirin is more likely to be misclassified as dying of cardiovascular diseases (CVD) is unfounded. All patients in Norway treated for CRC undergo regular clinical controls and surveillance with CT-scan every 6 months for 5 years to determine signs of recurrence or metastases, thus relapsed CRC almost never goes unnoticed. The suggestion that aspirin users are healthier or selected, meaning that 
they are less likely to die of CVD than the non-aspirin users is entirely based on speculation. Unfortunately, it is not possible to control for healthy user bias as we do not have data to characterize health-seeking or healthy life style behavior. However, if the aspirin users were more health-conscious, this would only counteract the effect that leads to the alleged misclassification of death as proposed by Løberg et al. We thus disagree with their unfounded claim that CRC-specific mortality is an invalid end-point. Our observation that CSS is comparable for all AJCC stages of CRC further supports the fact that misclassified cause-ofdeath does not differ significantly between the aspirin users and non-users [1]. Furthermore, analysis of the validity of cancer as cause of death supports use of CSS as an end-point [4].

The two meta-analyses referred by Løberg et al. support our conclusion that post-diagnosis aspirin use increases OS in CRC patients. However, they did not find a significant reduction in cause-specific mortality. Interestingly, the meta-analyses included almost the same primary publications (6 of 8 studies overlap in [5] and [6]) but report somewhat different conclusions. The aggregated cohort size in the meta-analyses is 30,397 patients for OS and 10,923 for CSS, the latter less than half the number of patients in our report as only some of the studies included CSS as primary endpoint. Among five primary studies that have analyzed CSS, four found a significant effect of aspirin [7-10] (Table 1).

In summary, we disagree with the speculations by Løberg et al. and think that it is an accumulated effect of aspirin use that provides potential patient benefit in the secondary preventive setting and not only use prior to diagnosis. 


\section{References}

[1] Bains S.J., Mahic M., Myklebust T.A. et al. Aspirin As Secondary Prevention in Patients With Colorectal Cancer: An Unselected Population-Based Study. J Clin Oncol 34:2501-8, 2016

[2] Løberg M., Holme Ø., Kalager M. Aspirin, colorectal cancer and cause of death - a complex landscape. J Clin Oncol, 2016

[3] Andersen P.K., Geskus R.B., de Witte T. et al. Competing risks in epidemiology: possibilities and pitfalls. Int J Epidemiol 41:861-70, 2012

[4] Skyrud K.D., Bray F., Møller B. A comparison of relative and cause-specific survival by cancer site, age and time since diagnosis. Int J Cancer 135:196-203, 2014

[5] Ye X.F., Wang J., Shi W.T. et al. Relationship between aspirin use after diagnosis of colorectal cancer and patient survival: a meta-analysis of observational studies. Br J Cancer 111:2172-9, 2014

[6] Li P., Wu H., Zhang H. et al. Aspirin use after diagnosis but not prediagnosis improves established colorectal cancer survival: a meta-analysis. Gut 64:1419-25, 2015

[7] Chan A.T., Ogino S., Fuchs C.S. Aspirin use and survival after diagnosis of colorectal cancer. JAMA 302:649-58, 2009

[8] McCowan C., Munro A.J., Donnan P.T. et al. Use of aspirin post-diagnosis in a cohort of patients with colorectal cancer and its association with all-cause and colorectal cancer specific mortality. Eur J Cancer 49:1049-57, 2013

[9] Liao X., Lochhead P., Nishihara R. et al. Aspirin use, tumor PIK3CA mutation, and colorectal-cancer survival. N Engl J Med; 367:1596-606,; 2012

[10] Domingo E., Church, D.N., Sieber O. et al. Evaluation of PIK3CA Mutation As a Predictor of Benefit From Nonsteroidal Anti-Inflammatory Drug Therapy in Colorectal Cancer. J Clin Oncol 31:4297-4305, 2013 
Table 1: Overview of studies of post-diagnosis aspirin use included in meta-analyses [5] and [6] ${ }^{\mathrm{a}}$

\begin{tabular}{|c|c|c|c|c|c|}
\hline Year & Authors & $\mathbf{n}$ & Outcome & $\mathrm{HR} / \mathrm{OR}^{*} / \mathrm{RR}^{* *}$ & $95 \% \mathrm{Cl}$ \\
\hline \multicolumn{6}{|c|}{ Studies of post-diagnosis aspirin use included in meta-analyses by both Ye et al [5] and Li et al [6] } \\
\hline \multirow[t]{2}{*}{2009} & Chan et al. & 1279 & OS & 0.79 & $0.65-0.97$ \\
\hline & & & CSS & 0.71 & $0.53-0.95$ \\
\hline 2012 & Bastiaannet et al. & 4481 & OS & $0.77^{* *}$ & $0.63-0.95$ \\
\hline 2012 & Walker et al. & 13994 & OS & 0.91 & $0.82-1.00$ \\
\hline \multirow[t]{2}{*}{2013} & McCowan et al. & 2990 & os & 0.67 & $0.57-0.79$ \\
\hline & & & CSS & 0.58 & $0.45-0.75$ \\
\hline \multirow[t]{2}{*}{2014} & Cardwell et al. & 4794 & OS & $1,06 *$ & $0.94-1.19$ \\
\hline & & & CSS & $1,06^{*}$ & $0.92-1.24$ \\
\hline 2014 & Reimers et al. & 999 & OS & $0.53 * *$ & $0.38-0.74$ \\
\hline \multicolumn{6}{|c|}{ Study of post-diagnosis aspirin use included in meta-analysis by Ye et al [5] only } \\
\hline \multirow[t]{2}{*}{2012} & Liao et al. & 964 & OS & $0.18^{\mathrm{b}}$ & $0.06-0.61$ \\
\hline & & & CSS & $0.54^{\mathrm{b}}$ & $0.31-0.94$ \\
\hline
\end{tabular}

Study of post-diagnosis aspirin use included in meta-analysis by Li et al [6] only

\begin{tabular}{|c|c|c|c|c|c|}
\hline \multirow[t]{2}{*}{2013} & \multirow[t]{2}{*}{ Domingo et al. } & \multirow[t]{2}{*}{896} & OS & $0.29^{b}$ & $0.04-2.31$ \\
\hline & & & CSS & $0.11^{b}$ & $0.001-0.832$ \\
\hline
\end{tabular}

\begin{tabular}{|c|c|c|c|c|c|}
\hline \multicolumn{6}{|c|}{ Study subject to discussion } \\
\hline 2016 & Bains et al. & 23162 & OS & 0.95 & $0.90-1.01$ \\
\hline & & & CSS & 0.85 & $0.79-0.92$ \\
\hline
\end{tabular}

OS, overall survival; CSS, cancer specific survival

a Space limitations prevents us from citing the primary reports, see [5] and [6] for references

${ }^{\mathrm{b}}$ In subset of patients with mutated-PI3K colorectal cancers 